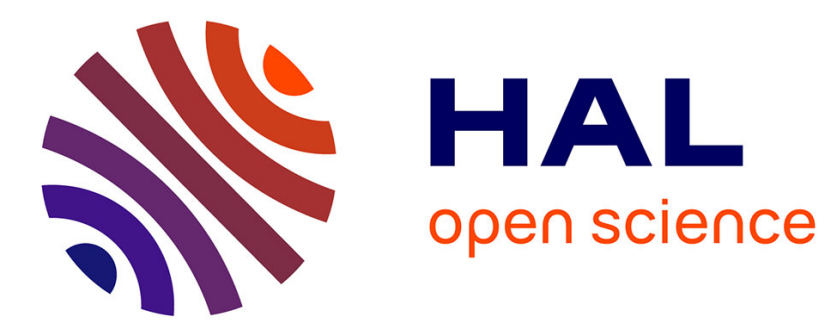

\title{
Veilleur, dormeur, somnambule : états du corps, états du moi biranien
}

\author{
Anne Devarieux
}

\section{To cite this version:}

Anne Devarieux. Veilleur, dormeur, somnambule: états du corps, états du moi biranien. Intellectica - La revue de l'Association pour la Recherche sur les sciences de la Cognition (ARCo), 2016, Phénoménologie de la vigilance et de l'attention. Philosophie, sciences et techniques, 66, pp.13-36. 10.3406/intel.2016.1814 . hal-02145258

\section{HAL Id: hal-02145258 https://hal.science/hal-02145258}

Submitted on 2 Jun 2019

HAL is a multi-disciplinary open access archive for the deposit and dissemination of scientific research documents, whether they are published or not. The documents may come from teaching and research institutions in France or abroad, or from public or private research centers.
L'archive ouverte pluridisciplinaire HAL, est destinée au dépôt et à la diffusion de documents scientifiques de niveau recherche, publiés ou non, émanant des établissements d'enseignement et de recherche français ou étrangers, des laboratoires publics ou privés. 


\title{
Veilleur, dormeur, somnambule : états du corps, états du moi biranien
}

\author{
Il y a en effet bien des manières de dormir, et tout autant qu'il y a des manières de \\ veiller...
}

\section{Titre de l'article en anglais:}

Awake, asleep, sleepwalking - States of the body, states of the self in Maine de Biran's thought.

\section{Résumé en français}

Dès le Mémoire sur la décomposition de la pensée, Maine de Biran assimile la tension immanente et générale de l'effort à la veille du moi. L'individu n'acquiert la conscience de soi que par la tension de sa volonté qui est identiquement la tension de son corps résistant, et cela indépendamment de toute visée intentionnelle expresse, de toute perception ou représentation active d'objets (effort dit intentionné ou express).Il faut donc distinguer des degrés variables de l'effort, depuis l'aperception pure (dans laquelle l'ego apparaît comme un veilleur) en passant par l'attention (qui est une vigilance accrue et dirigée intentionnellement vers le dehors) jusqu'à la réflexion (qui représente le plus haut degré de tension). Par conséquent l'effort (ou la dualité interne formée par le couple de la force dite hyper-organique et de la résistance intérieure du corps propre) constitue l'aperception immédiate interne, et varie seulement en degrés d'intensité. Le sommeil est selon Biran la suspension périodique du vouloir et, avec lui, de tout sentiment de soi. Le moi renoue pourtant avec lui-même au réveil dès lors qu'une même force se déploie sur une même résistance.

Il y a pourtant soulignera Biran bien des manières de veiller et de dormir, y compris à l'état de veille, et les analyses de Biran permettent de comprendre comment peuvent surgir dans nos vies conscientes des états hypnotiques voire somnambuliques qui ressemblent à s'y méprendre au sommeil (Biran commente notamment J.-P.F. Deleuze). La question est en définitive de saisir les enjeux de telles considérations dès lors que Biran refuse d'assimiler l'immédiateté de conscience (appropriation motrice) à l'immédiateté affective d'une vie quasi hypnotique à la manière par exemple du phénoménologue Michel Henry pourtant grandement influencé par sa pensée.

\section{Résumé en anglais :}

According to the philosopher Maine de Biran, in his Mémoire sur la décomposition de la pensée, the immanent and general tension of the effort is equal to the awakeness of the Ego. The individual gains self-consciousness thanks to the tension of his will, namely in the general tension of his resistant body, independently of any intentional aim, of any active perception or representation of objects (the so-called "intentionned" or "express" effort). Thus it is necessary to distinguish different degrees of effort, from pure self-apperception (in which the ego appears like a watchman) to attention (increased vigilance of the will intentionally tensioned outwards) and reflection (the

\footnotetext{
${ }^{1}$ Biran, 1955, p. 422.
} 
highest degree of alertness). Consequently the effort (that is the internal duality composed by two terms, the so-called "hyper-organic" strength and the inner resistance of the muscular system of the body) constitutes the immediate and internal apperception of the Ego, and varies according to its own intensity. Sleep is, according to Biran, a periodical suspension of the will and consequently of the sentiment of effort : in such a state, self-consciousness vanishes. Nevertheless upon awakening, the person once again becomes self-conscious, since the same strenghth meets the same body's resistance.

So there are many ways of being awake, many ways of sleeping, even during the day-time, and Biran helps us to understand how hypnotic and indeed even somnambulistic states, not far at all from sleep, can take place, in our conscious lives (Biran reads and comments in particular the writings of J.-P.F. Deleuze). The question is ultimately to apprehend the full implications of such considerations since Biran refuses to assimilate self-consciousness (motor activity) to some kind of hypnotic and purely affective life (as in Michel Henry's phenomenological analysis, and despite his acknowledged debt to Maine de Biran's thought).

Mots-clés: Veille, Agentivité, Conscience de soi, Sommeil, Somnambulisme, Aperception, Attention, Réflexion, Effort, Hypnose, Affectibilité, Force hyperorganique, Résistance intérieure du corps propre, Vigilance.

Key-words : Awakeness, Agency, Awareness, Sleep, Somnambulism, Apperception, Attention, Reflection, Effort, Hypnotism, Affectivity, Hyper-organic strength, Inner resistance of one's own body, Alertness.

\section{Introduction}

Nous nous proposons, dans le cadre de cet article, d'introduire à la compréhension du lien forgé par le philosophe français Maine de Biran ${ }^{2}$ entre la conscience de soi et le sentiment d'effort,

\footnotetext{
${ }^{2}$ Maine de Biran né en 1766 à Bergerac-mort en 1824 à Paris. Philosophe et homme politique français : élu au conseil des Cinq-Cents par l'assemblée préfectorale de la Dordogne (1797), membre du conseil général de la Dordogne (1802), sous-préfet de l'arrondissement de Bergerac (1806), membre du corps législatif (1810), député à Paris, membre de la « Commission des Cinq » (1813), questeur de la Chambre (1814), Conseiller d'Etat au comité de l'Intérieur (1816). Il a publié de son vivant trois écrits: Influence de l'habitude sur la faculté de penser, Examen des Leçons de philosophie de M. Laromiguière, et l'Exposition de la doctrine philosophique de Leibniz. Son œuvre est publié chez Vrin (CEuvres, Vrin, Paris, dir. F. Azouvi : 1984-1999) et comprend outre les ouvrages achevés, des articles, des réflexions politiques, des notes philosophiques, et une correspondance très riche. Il est aussi l'auteur d'un Journal. D'abord idéologue (proche notamment de Destutt de Tracy et de Cabanis) Maine de Biran « devient biranien » en rédigeant le Mémoire sur la décomposition de la pensée. Philosophe de l'effort, il est le premier à introduire le corps propre dans la définition du fait primitif de conscience qui relève d'une certitude non représentative. Métaphysicien de l'expérience intérieure,
} 
de faire entendre la raison de l'assimilation faite dès le Mémoire sur la décomposition de la pensée entre la tension immanente et générale de l'effort et la veille du moi. L'individu est cette conscience vigile, donnée à elle-même, dans la tension de son vouloir (effort dit « immanent » ou « général ») qui est identiquement la tension générale de son corps résistant, indépendamment de toute visée intentionnée, de toute perception active d'objet (effort dit «intentionné » ou « exprès »). Biran distingue l'état de veille de "ce degré de l'effort supérieur" qu'est selon lui l'attention laquelle relève d'une "volonté positive et expresse". L'effort propre à l'aperception immédiate interne, va par degrés dans l'état de veille, et le sommeil est la suspension périodique du vouloir et, avec lui, de tout sentiment de soi. Le moi renoue pourtant avec lui-même au réveil dès lors qu'une même force se déploie sur une même résistance.

Tandis que le sentiment d'effort est uniforme, constant, à travers la succession de ses modes, les affections de toutes sortes sont extrêmement variables. Chacun de ces deux éléments a donc sa marque propre, sur laquelle il est impossible de se tromper. Dans la vie normale de l'homme, ils se combinent en proportions différentes; mais l'animal vit d'une vie purement affective, et chez les hommes mêmes, il y a une multitude d'états, tels que le sommeil, le somnambulisme, la catalepsie, la démence, qui restent en dehors de la conscience proprement dite.

Il y a par conséquent bien des manières de veiller et de dormir, - l'homme assoupi à l'état de veille ressemble à s'y méprendre à l'homme éveillé - et apparaît comme problématique l'irruption dans la vie éveillée d'états quasi somnambuliques voire hypnotiques que le philosophe analyse, en les distinguant du sommeil proprement dit. Ces considérations aboutissent à l'hypothèse biranienne quelque peu surprenante de deux moi. La question est de savoir ce qui se noue dans ces analyses dès lors que Biran refuse d'assimiler l'immédiateté de conscience, ou l'appropriation motrice à l'immédiateté affective d'une vie quasi hypnotique à la manière par exemple du phénoménologue Michel Henry. Nous procédons à l'examen en trois temps: la veille, le sommeil, et le somnambulisme.

\section{VEILLER}

Parti à la recherche de ce qui nous donne le sentiment de nous-mêmes, Maine de Biran découvre, dès son premier Mémoire (Influence de l'habitude sur la faculté de penser), le sens de l'effort comme une relation primitive, tout intérieure et duelle, mettant en jeu deux termes, une force dite hyper-organique ${ }^{3}$ et la résistance du corps propre. Mais il faut attendre le Mémoire sur la

\footnotetext{
phénoménologue avant la lettre (cf. les lectures de Merleau-Ponty, Jan Patočka, ou Michel Henry), « le plus grand métaphysicien que la France ait produit depuis Descartes ou Malebranche » selon Henri Bergson.

${ }^{3}$ Est "hyper-organique" ce qui ne se donne que par conscience. On trouve le terme "hyper-organique" dans les Principes de physiologie de C.-L. Dumas mais sa signification est tout autre que celle que lui donne Biran plutôt inspiré par les analyses de Marie François Xavier Bichat (voir F. Azouvi, 2000, p. 83).
} 
Décomposition de la Pensée pour que soit définitivement formulée la dualité intérieure primitive et qu'elle soit assimilée à l'état de veille du moi:

Le principe de la vie de relation et de conscience une fois en activité et en pleine possession de son domaine, se reproduit constamment sous une même forme. Son exercice périodiquement suspendu, pendant que celui de la vie absolue ne s'interrompt point, il renait toujours égal ; c'est toujours la même force qui lutte contre le même terme résistant, il ne peut y avoir là de variations que dans le degré. La veille du moi est constituée par cet état d'effort immanent. Tant qu'elle subsiste la personne est plus ou moins mais toujours identiquement présente à elle-même ${ }^{4}$.

Loin de désigner le simple fait d'être éveillé, la veille $d u$ moi relève de l'activité par laquelle l'individu entre en possession de lui-même, devient dans une certaine mesure maître de soi, compos sui, conscium sui (aime à dire Biran), bref capable de s'apercevoir lui-même. Veiller, c'est par conséquent monter la garde et c'est ainsi qu'apparaît en effet l'individu biranien : comme un être aux aguets, vigilant. Souligner l'immanence de l'effort propre à notre état de veille, c'est aussi pour le philosophe montrer à quelles conditions l'on est «à soi ou en soi » ${ }^{5}$ et non pas « hors de soi », distrait de soi, aliéné. Mode fondamental de notre existence vigile, celle de notre vie de relation, l'effort immanent qui seul fait notre veille, est d'intensité variable et son exercice périodiquement suspendu.

Avant de revenir sur cette double caractéristique, remarquons d'une part que l'aperception de soi n'est pas dans la sensation de mouvement chère à Destutt de Tracy, mais bien dans le sentiment de notre activité, - le cogito est un volo - le sentiment d'être l'auteur des actes que nous déterminons comme moteur : «motilité de conscience ${ }^{6}$. Il n'est pas d'autre part défini par l'attention que l'individu prêterait au monde ou à lui même. La veille du moi est en effet un effort sans effort apparent, c'est-à-dire sans acte d'attention intentionné ou exprès : il est effort « général » mobilisant la masse du corps résistant. Enfin le moi n'est pas constitué en un rapport de dépendance avec le monde extérieur : il est d'abord donné à lui-même, et peut dès lors s'ouvrir au monde ${ }^{7}$.

Semblable à un veilleur de nuit, toujours aux aguets dans la pénombre du monde ${ }^{8}$, en l'absence de lumière, sa vigilance ou active surveillance est littéralement sans objet - pour la même raison que «le sens intime n'a pas d'objet ${ }^{9}$, c'est-à-dire qu'il ne relève pas de la certitude représentative - et n'est autre que la mobilisation active, la tension générale de l'étendue intérieure

\footnotetext{
${ }^{4}$ Biran, 1988, p. 141.

${ }^{5}$ Biran, 2001, p. 3.

${ }^{6}$ Biran, 1996, p. 328. C'est un hapax.

${ }^{7}$ Une telle analyse retiendra toute l'attention de Michel Henry (1965). A savoir l'affirmation d'une conscience sans monde, la dualité vie/monde, l'affirmation d'un effort non intentionnel, nerf de la critique henryenne de l'intentionnalité husserlienne. La reprise biranienne très singulière dans l'œuvre de Michel Henry ne peut évidemment être analysée dans le cadre de cet article : elle est l'objet d'un livre à paraître.

${ }^{8}$ CF. Xavier Tilliette, 1983, p. 440.

${ }^{9}$ Biran, 1996, p. 240.
} 
continûment résistante de son corps. Un tel sentiment de soi relève d'une «nécessité de conscience » ou d'une nécessité de sentiment, bien distincte d'une nécessité logique ou rationnelle. L'autre nom d'un tel mouvement de conscience par lequel l'individu est donné à lui-même est celui de volonté dont les limites ne sont autres que celles de son pouvoir ${ }^{10}$. Le Mémoire sur la décomposition énonce ainsi la question :

Chercher [...] la condition commune qui, servant à effectuer dans l'origine le déploiement d'une force vivante, sert aussi à circonscrire dans son domaine propre, et par autant d'actes relatifs de conscience, les termes multiples de son application, à distinguer ainsi, dans leurs limites réciproques, les termes inertes qui obéissent à la même puissance motrice, et ceux qui résistent sans obéir. ${ }^{11}$

Biran trouve confirmation de la nécessité d'une influence motrice dans les expériences menées par le médecin de Montpellier M. Rey Régis : en cas de paralysie, le malade à qui l'on touche une partie du corps, éprouve un sentiment diffus qu'il est dans l'incapacité de localiser : «le rapport direct des impressions à un siège tiendrait donc originairement aux mêmes conditions que la motilité volontaire $»^{12}$.

Mon individualité est par conséquent à la croisée ou au point de rencontre entre une même force qui s'exerce sur une même résistance: la veille est cet état de tension générale, tonos immanent et inintentionné. Le Mémoire de Berlin le condensera en formules remarquables :

Pour concevoir ce moi phénoménal dans le sens unique et individuel de son aperception immédiate, séparée de tout ce qui n'est pas elle, supposons tous les muscles volontaires contractés dans l'immobilité du corps, les yeux ouverts dans les ténèbres, l'ouïe tendue (acuta) dans le silence de la nature, l'air ambiant en repos et la température extérieure en équilibre avec celle de la surface du corps, toutes les impressions internes réduites au ton naturel de la vie organique, insensibles dans leur continue uniformité...l'effort reste seul, et avec lui, le moi phénoménal pur ou réduit à son aperception immédiate interne; tant que ce mode invariable persiste, c'est-à-dire tant que dure la veille du moi, les impressions sensibles et accidentelles, coïncidant avec lui, peuvent participer suivant diverses lois ou conditions à son activité reproductive et à la lumière de conscience qui jaillit de cette source ${ }^{13}$.

\footnotetext{
${ }^{10}$ «Et si tous les termes partiels, ou le corps en masse était dans le même état, toute aperception ne se trouverait-elle pas complètement suspendue, comme dans le sommeil, quoique l'affectibilité passive pût subsister ? Le rapport direct des impressions à un siège tiendrait donc originairement aux mêmes conditions que la motilité volontaire ; il pourrait donc être séparé de la partie affective » (Biran, 1988, note p. 140). On peut rapprocher les analyses biraniennes de la notion contemporaine d'agentivité. De même, l'existence du locked-in Syndrome semble donner raison à Biran lorsqu'il considère que la paralysie entraine l'absence d'aperception ou de sentiment de la partie concernée : le patient ne communiquant qu'à l'aide des seuls mouvements volontaires restant à sa disposition (ouverture des yeux, clignement des paumières).

${ }^{11}$ Biran, 1988, p. 139.

${ }^{12}$ Ibid, p. 140.

${ }^{13}$ Biran, 1995, p. 137-138.
} 
Cette description, dense et resserrée, - proche d'une réduction phénoménologique ${ }^{14}-\mathrm{du}$ phénomène de l'aperception de soi livre le moi à l'état de pureté, d'un individu bandé, aux aguets, dans la pénombre, dont tous les muscles sont tendus et les sens activés, mais en dehors de toute perception réelle, un être tendu en pure perte pourrait on dire : dans la nuit - le monde et sa lumière sont mis entre parenthèses -, l'individu, bien que plongé dans les ténèbres, a les yeux pourtant grand ouverts, l'ouïe tendue par la force hyper-organique du vouloir, bref tous ses sens - ou ce qu'on appelle tels - sont en éveil, tandis que la force vitale aveugle que Biran distingue en nature de l'effort, fait, comme d'habitude son jeu, inlassablement. A cette pure aperception de soi les impressions affectives et les intuitions ou représentations - en la compliquant - s'agrègent, s'unissent de façon diverse et inégale mais sans jamais constituer l'effort proprement dit. Voilà l'état de veille. Ne jurerait-on pas une description de l'insomnie?

Bien que la force vitale donnant le ton de la vie organique soit d'un autre ordre pour Biran que la force dite hyper-organique (Biran ne la nomme pas spirituelle non plus que matérielle) s'exerçant contre la résistance intérieure du corps, soulignons pourtant que Biran emprunte ici à G.E. Stahl son vocabulaire (servant à définir le principe vital) pour formuler le «moi phénoménal ${ }^{15}$, en même temps qu'il le critique ouvertement pour avoir étendu l'activité de l'âme (dont il n'a pourtant pu trouver l'idée que dans l'aperception personnelle) à toute activité organique sans avoir fait par conséquent comme il le faudrait, la discrimination entre les mouvements organiques non aperçus, les mouvements sentis en résultats sans volonté expresse, et enfin les mouvements sentis et volontaires.

Pour qu'il y ait sentiment de soi, il faut en effet un corps résistant qui réplique à une force. Il y a donc une sphère d'exercice de l'effort, car tout dans mon corps n'est pas résistant : il y a des affections dites pour cette raison « pures », proprement « illocalisables », car purement affectives et à ce titre non aperçues, non senties sur le continuum résistant. Il faut donc discriminer entre nos mouvements comme entre nos sentiments : l'effort de l'individu motile dans l'immobilité du corps mobilisé en masse s'exerce d'abord en deçà de toute circonscription de ses parties. La vigilance est l'état de veille, qui est nous-même. Quand la veille par conséquent s'interrompt et que vient (quand il vient) le sommeil, alors nécessairement (et pour notre plus grand étonnement) disparaît le sentiment de soi.

Insistons : une telle découverte suppose en amont et en aval la discrimination entre nos mouvements car la volonté ne relève pas d'une force motrice qui serait innée à une âme substantielle ; elle n'est pas davantage dans les déterminations de l'instinct, ni dans les mouvements du fœtus ou de l'enfant, ou ceux aveugles de l'habitude, pas plus que dans ceux du somnambule. Autrement dit, la volonté n'est rien hors du sentiment de l'activité; il y a donc des mouvements sans effort, sans aperception; et le mouvement produit dans l'extériorité, visible, soit le résultat du mouvement de conscience n'est pas le mouvement primitif de conscience tel que l'entend Biran : ce dernier en effet n'est pas d'abord essentiellement un moyen, - même s'il l'est aussi secondairement

\footnotetext{
14 «Toute l'œuvre de Biran n'est qu'une vaste réduction phénoménologique » (Michel Henry, 1965, p. 25).

${ }^{15}$ La force « veille comme une sentinelle vraiment active... » (Biran, 2001, p. 264).
} 
- ni plus ni moins que la vigilance n'est d'abord tournée vers le monde. Se lever pour faire la lumière dans le noir, voilà le résultat d'un même effort (immanent) toujours disponible - il est la disponibilité même à soi - tant que dure l'état de veille ou le sentiment individuel de mon existence propre par lequel je suis donné à moi-même. La veille est inséparable du sentiment et pressentiment $^{16}$ de l'énergie disponible d'un pouvoir (et non pas de la connaissance objective des moyens de l'action), elle fait le « durable du moi », pour reprendre l'expression biranienne, à savoir toute la durée de ma vie consciente.

Ce mode fondamental en deçà duquel on ne peut par conséquent régresser, et qu'on ne peut davantage expliquer (il s'agit pour nous de le gagner, d'élever son sentiment à la hauteur d'une idée), ne varie qu'en degré, tout relatif qu'il est à l'énergie du vouloir : il est une force intensive. Mais en tant qu'effort immanent, l'individu est donné à lui-même complètement, définitivement : la trace de l'effort fluant uniformément constitue le sentiment de l'identité personnelle. Or les variations d'intensité de ce même effort vont de pair avec la visée vers le monde et/ou le retour à soi (« attention » et « réflexion » dans le vocabulaire de Biran).

C'est l'Essai sur les fondements de la psychologie qui définit en effet deux modes de l'effort qu'il faut distinguer : l'un immanent et général, l'autre intentionné. Le Mémoire de Berlin comme le Mémoire sur la décomposition de la pensée formulaient certes déjà l'état de veille, mais ne distinguaient pas expressément ces deux modes de l'effort (bien qu'ils contenaient tous deux des analyses similaires de ce que sont l'aperception, l'attention, et la réflexion) :

Il importe d'avoir présente ici une distinction que nous avons déjà établie d'une manière générale, entre deux modes d'exercice de l'effort qui diffèrent l'un de l'autre, non par le principe ou la cause une, mais par les résultats ou les effets. En vertu du premier mode, tous les organes sur lesquels la volonté peut agir ou qui font partie du sens de l'effort commun, sont rendus aptes à percevoir leurs objets propres, quoiqu'il n'y ait point de perception actuelle. Cet effort non intentionné, qui s'étend à tous les muscles volontaires, constitue, avec le durable du moi ou de la personne identique, l'état de veille de ces sens divers qui concourent à la vie de relation ou de conscience. C'est ainsi que le sens de la vue veille dans les ténèbres, usque in spissi tenebris, celui du toucher, hors de toute pression accidentelle, celui de l'ouïe dans le silence etc. Périodiquement suspendu pendant le sommeil, l'exercice de la volonté, tandis que la vie organique roule sans interruption dans son cercle accoutumé, redevient présent à lui-même, dès que la même force recommence à se déployer sur la même inertie organique. Le sujet de l'effort reconnaît immédiatement son identité, sa durée continuée et sent qu'il est le même

\footnotetext{
${ }^{16}$ Voir notamment les critiques adressées à Hume (Biran, 1993, p. 37 et sq). A ce titre l'effort n'est pas un « faire effort » mais, identique à l'état de veille, il désigne le sentiment d'effort uniforme, constant, le sens intime lui-même. Une même force est en butte au même terme résistant, telle est la mobilisation de tous les muscles volontaires donnant naissance à l'étendue intérieure continûment résistante (Biran, 1988, p. 432) qui assure la « continuité de moi ». Michel Henry évoque à ce propos non sans légitimité la « sorte de tension latente avec laquelle se confond l'être même de notre corps absolu et qui retient pour ainsi dire, dans l'unité de sa propre vie, notre corps organique et peut-être l'effectivité de toute présence au monde en général » (Henry, 1965, p. 230).
} 
qu'avant le sommeil, sans qu'aucune impression accidentelle vienne motiver des souvenirs distincts, ou quelque relation déterminée entre un temps présent et un temps passé. ${ }^{17}$

Et plus loin Biran précise :

Il y a, comme nous l'avons vu, un effort commun qui s'exerce simultanément dans l'état de veille sur tous les organes qui rentrent dans la sphère d'activité de la force une. C'est cette force qui, comme le dit si énergiquement Stahl, veille comme une sentinelle vraiment active sur les organes de la sensibilité : organis sensoriis vere active excubias agit. Cet exercice général et continu de l'effort, qui fait la conscience ou le durable de notre existence personnelle, est le lien premier et nécessaire de l'union du moi avec les modes variables (affectifs ou intuitifs) qui coïncident avec l'effort et se coordonnent par lui dans un temps ou un espace. ${ }^{18}$

L'effort immanent dessine et délimite par ses actes successifs l'étendue intérieure d'abord vague et illimitée du corps propre et le temps naît alors, s'il est vrai que le temps n'existe pour moi que par la trace laissée par l'effort seule apte à former le sentiment de continuité de soi. Ces deux modes de l'effort ont donc même principe mais non point même résultat; dans le premier mode, le mouvement de conscience, puisque mouvement il y a, est celui de l'activation des sens en pure perte, sans visée expresse : le moi est constitué, il s'aperçoit mais ne perçoit rien. Biran appelle en revanche effort intentionné ou exprès ce même mouvement en tant qu'il nous porte à un dehors qu'il vise ; tel est le rôle propre de ce que Biran appelle l'attention. C'est dire que la vigilance n'est pas l'attention, que moi est donné à lui-même sans mouvement intentionnel porté vers le monde.

Et c'est parce que les modes de notre existence peuvent soit participer accidentellement à l'aperception, soit s'y approprier en résultats alors qu'ils sont étrangers à son principe, soit enfin irradier de la source de l'effort et participer à tous ses caractères, que Biran peut distinguer trois classes de modifications: le système sensitif passif qui comprend les affections intérieures et extérieures sensibles, sans le concours de la volonté et du moi ; le système perceptif ou intuitif qui suppose une cause étrangère à la volonté, notre absence d'initiative volontaire mais la réaction de cette dernière (sensations composées, localisées), et enfin le système aperceptif actif qui remonte des produits immédiats et exclusifs du vouloir à leur principe soit l'effort.

Quel lien établir dès lors entre la vigilance du moi et l'attention ? Fidèle à sa méthode de décomposition, Biran qualifie différemment l'union du moi avec les modifications selon que la partie sensible, affective ou représentative, y est plus ou moins élevée. La «perception» proprement dite suppose en effet la prédominance de la partie représentative : ainsi de la vue, de l'ouïe et du toucher. Biran prend l'exemple de la vision activée par le vouloir, du regard activé par la volonté :

Lorsque la vision est en effet activée par un vouloir exprès (vivida intentione actuanturexpression énergique de Stahl) son organe prend une impression particulière, où reluit comme la vie et le feu pénétrant de l'intelligence (ignis intelligens); mais l'expression qui anime ainsi le regard ne vient point de l'organe même ; c'est une irradiation de la force de l'âme, dont l'œil

\footnotetext{
${ }^{17}$ Biran, 2001, p. 239.

${ }^{18}$ Ibid, p. 264.
} 
devient à son tour le miroir ; c'est cette force qui dirige le point visuel, le fixe tour à tour sur les différentes parties de l'objet, agite et tourne l'œil dans tous les sens, l'électrise par frottement ou communication, et lui donne ainsi toutes les conditions essentielles à une perceptibilité distincte ${ }^{19}$.

La perceptibilité suppose la part représentative de l'intuition, que l'attention peut reprendre en compte; les intuitions visuelles passives, hors effort, sont par Biran distinguées de l'attention qu'il définit comme « un exercice du sens de l'effort plus exprès et plus intentionnel, (qui) peut toujours dans le regard activé par le vouloir, élever l'intuition simple au rang de perception complète $»^{20}$. A l'inverse, la sensation passive peut absorber l'effort et ce dernier peut ne pas être aperçu, même s'il y a attention, dès lors que le sens externe de l'intuition prend l'initiative sur la volonté : l'effort n'est plus senti alors que dans son résultat médiat (non pas dans sa détermination propre ni dans le résultat immédiat), c'est-à-dire dans l'image sur lequel l'attention se concentre. En effet, l'intensité de l'effort augmente en fonction de la complication perceptive :

J'appelle attention ce degré de l'effort supérieur à celui qui constitue l'état de veille des divers sens externes, et les rend simplement aptes à percevoir ou à représenter confusément les objets qui viennent les frapper.$^{21}$

Telle est toute la différence entre l'attention et la réflexion qui s'attache aux actes propres $\mathrm{du}$ vouloir et non à leurs résultats modaux. Les modes de la pensée, ce que l'on nomme traditionnellement les «facultés » ne sont autres en effet que les produits actifs de la puissance individuelle et une d'effort. Une telle analyse permet de comprendre a contrario combien nous avons tendance à être captés ou fascinés par les résultats de nos actes, au détriment de la conscience de la force et de ses résultats immédiats (résistance immédiate interne du corps). La puissance de diversion de l'image est telle que l'effort est alors confondu alors avec son résultat modal, et Biran rapporte au caractère prédominant de la vue (chez l'homme) cette puissance de l'image et de l'imagination. De la même façon, le philosophe fait la différence entre l'audition passive et l'auscultation, entre le tact passif et le toucher actif, etc. Dans le système aperceptif, le rôle prédominant revient au toucher actif : si nous ne sommes ni plus ni moins certains de nous-même que des corps extérieurs, c'est que dans les deux cas, la volonté ici commence et continue le mouvement : l'aperception immédiate interne et externe l'emporte sur la simple perception et la réflexion «concentrée » dans l'acte l'emporte sur l'attention qui suit au dehors les résultats de l'acte.

Résumons : le propre de l'attention est d'exclure la passivité complète, mais parce qu'elle est focalisée sur son résultat, elle est dite moins éminemment active que la réflexion ${ }^{22}$. Vigilance pure (aperception de soi), vigilance expresse (attention), redoublement ou repliement de

\footnotetext{
${ }^{19}$ Biran, 1995, p. 146.

${ }^{20}$ Ibid, p. 147.

${ }^{21}$ Biran, 2001, p. 265.

${ }^{22}$ Soulignons que Michel Henry mettra en question une telle précision.
} 
vigilance (réflexion), voilà, pourrait-on dire, les trois modes de la veille dont l'intensité va croissante.

On voit tout le rôle dévolu au mouvement par Biran : ce sont les mouvements volontaires qui, associés avec les intuitions, en deviennent les signes disponibles ${ }^{23}$. Dans l'attention, le vouloir est en tout cas arrêté sur le résultat de l'action, au détriment de l'aperception du principe. Autant dire que le résultat d'un acte ou son produit perceptif est par sa nature même d'objet un mobile de distraction (le vouloir y est distrait de son principe) tout comme il est (et paradoxalement) le produit d'une activité volontaire, d'une intensité supérieure à l'effort général. Biran écrit dans l'Essai que toute la différence entre les «mouvements produits sans conscience actuelle quoique déterminés antérieurement par une volonté expresse » et les actes d'aperception «consiste en ce qu'il y a distraction dans le faible déploiement de la force motrice et attention dans son exercice plus intense $\iota^{24}$.

Ou encore :

Le degré supérieur dont il s'agit est déterminé par une volonté positive et expresse qui s'applique à rendre plus distincte une perception d'abord confuse, en l'isolant, pour ainsi dire, de toutes les impressions collatérales qui tendent à l'obscurcir ${ }^{25}$.

Ainsi le pouvoir de l'attention consiste à fixer les organes mobiles à volonté comme l'ouïe et le toucher sur l'objet présent. Penser dans ces termes la veille du moi, comme une surveillance active et sans objet par laquelle l'individu est donné à lui-même, c'est identiquement penser la possibilité même de la distraction inhérente à l'état de veille : l'effort est en effet enveloppé d'abord par la vie elle même - notre première habitude étant de vivre ${ }^{26}$ - et par les produits médiats de notre effort. C'est dire que nous devons gagner ou regagner le sens de nous-même. Et la réflexion aura précisément pour tâche de porter à l'idée ce dont l'aperception nous donne le sentiment : c'est le sens de l'ouïe couplé à la voix qui en fournit le modèle ${ }^{27}$.

Biran nomme précisément « effets de représentation » les produits ou résultats de l'effort non aperçu sinon dans ses résultats, ceux-là même qui impliquent de notre part distraction, diversion. Il y a en effet bien des mouvements et par conséquent bien des manières de veiller : des résultats du mouvement primitif, internes ou externes, mais aussi des « effets » purs pour ainsi dire, qui ne sont en rien des mouvements volontaires. Tous les résultats de nos actes sont par conséquent en droit séparables de leur source active, et tout à la fois peuvent provoquer en résultat une appropriation. C'est ainsi que Biran tente de penser le passage du sommeil au réveil, au retour

\footnotetext{
${ }^{23}$ «L'attention ne s'applique donc spécialement qu'aux sensations représentatives déjà coordonnées dans l'espace et le temps, et ayant pour siège des organes qui rentrent dans le sens de l'effort, tandis qu'elle n'exerce aucune influence directe sur les impressions affectives, qui s'exaltent ou s'obscurcissent d'elles-mêmes suivant les lois d'une sensibilité spontanée » (Biran, 2001, p. 266).

${ }^{24}$ Biran, 1988, note p. 418.

${ }^{25}$ Biran, 2001, p. 265.

${ }^{26}$ La vie est l'irréfléchi même. Cf. Biran, 1987.

${ }^{27}$ Sur ce point, Anne Devarieux, 2004.
} 
périodique de l'éveil. Le déploiement de la force hyper-organique, bien que distincte de l'énergie vitale suppose des conditions physiologiques d'aperceptibilité, que Biran tente de retracer en différenciant les mouvements instinctifs puis spontanés et les mouvements proprement volontaires. Mais le rapport essentiel et vraiment primitif de causalité ne diffère pas de l'existence même : il est notre vie de relation, et suppose une sphère d'exercice qui circonscrit le domaine de la puissance d'effort. $^{28}$

\section{Dormir}

Tandis que le mouvement de la vie est incessant, le mouvement de l'effort est périodiquement suspendu et renait. Comment, après Buffon, ne pas s'en étonner ? ${ }^{29}$ Dormir, c'est fondamentalement s'absenter non du monde, mais de soi. C'est se déprendre de soi. Certes à l'état de veille il y a des impressions non senties intérieures, hors effort (nous sommes, pour ainsi dire, des «paralytiques » de la sensibilité intérieure) ; mais il s'agit désormais de comprendre ces deux modes alternatifs d'existence que tout éloigne.

Et de rassurer le «premier homme » de Buffon : «Il n'a rien perdu dans le sommeil, et il retrouve toute son existence, son moi tout entier, dès qu'il recommence à agir et vouloir ${ }^{30}$. Que notre existence fasse alterner ces deux modes n'implique pas en effet qu'il faille, selon la conclusion qu'en tirera Michel Henry, s'imaginer un moi absent et qui renaîtrait de façon incompréhensible toutes les fois qu'il s'efforce ${ }^{31}$. Nous l'avons vu, à l'état de veille, en l'absence de tout acte de perception objective, le moi est donné à lui-même dans une tension immanente ; sa suspension périodique pendant le sommeil n'empêche sa renaissance à lui-même au réveil, dès lors que la même force rencontre la même résistance. Le sommeil est le relâchement de la tension de la force active, absence du soi à lui-même. Il dépend donc de nous, à l'état de veille, - telle est l'œuvre propre de la réflexion - de réactualiser ce sentiment contre l'habitude qui œuvre à transformer l'aperceptibilité de droit de nos mouvements en impossibilité de fait. Mais comment comprendre à la fois cette suspension et le réveil ou éveil de la énième fois ?

Si le moi se retrouve au réveil, s'il met pour ainsi dire la main sur lui et sur personne d'autre, c'est que la même résistance se heurte à la même force ! En termes ricœuriens, la mêmeté

\footnotetext{
${ }^{28}$ L'expérience du médecin de Montpellier Rey-Régis vient aux yeux de Biran confirmer ses propres dires, à savoir que la paralysie entraine, avec l'absence d'aperception, l'impossibilité de localiser la sensation (Biran, 1988, note p. 139140).

${ }^{29}$ « Dans un monologue admirable par la sublimité de l'expression autant que par la profondeur des pensées, l'immortel Buffon fait dire au premier homme, quand il se rend compte à lui-même des effets de ses nouvelles impressions, et au sujet du sommeil dont il goûte les douceurs pour la première fois : «Tout fut effacé, tout disparut : la trace de mes pensées fut interrompue; je perdis le sentiment de mon existence. Ce sommeil fut profond. Mon réveil ne fut qu'une seconde naissance, et je sentis seulement que j'avais cessé d'être. Cet anéantissement que je venais d'éprouver me donna quelque idée de crainte, et me fit sentir que je ne devais pas exister toujours [...] ». Biran, 1984, p. 83.

${ }^{30}$ Ibid.

${ }^{31}$ De ce point de vue la critique henryenne de la passivité biranienne (Henry, 1965) nous semble porter à faux.
} 
des deux termes de la relation, garantit l'ipséité. ${ }^{32}$ Mais comment comprendre et formuler le passage de l'un à l'autre ? si l'on examine le second, soit le passage du sommeil au réveil, Biran essaie de l'expliquer, en distinguant notamment à la suite de Bichat, la contractilité organique, animale et volontaire, mais hors même de toute explication physiologique - lesquelles sont pour Biran toujours symboliques et n'atteignent pas le signifié lui-même -, le sens intime nous permet de faire la discrimination entre nos mouvements et d'expliquer le passage de nos mouvements spontanés aux mouvements proprement volontaires. Biran fait la description de ce qu'on appelle réveil :

Dans le sommeil de la pensée ou du moi, par exemple, il nous arrive de nous réveiller tout à coup par des mouvements, des paroles ou des voix, tous produits d'une spontanéité semblable à celle qui venait dans l'origine se placer entre l'instinct et la volonté. En se réveillant, l'individu sent ses mouvements non pas accompagnés de l'effort ainsi que dans l'état de veille, mais bien comme pouvant les faire et les répéter librement, il semble dans ce cas que le moi qui a le souvenir de son effort précédent s'approprie en résultat des actes qu'il n'a pas déterminés en principe et cette appropriation de conscience caractérise seule le réveil complet ${ }^{33}$.

C'est donc le sentiment et par conséquent le souvenir du pouvoir exercé qui caractérise le réveil du moi : le pressentiment et l'aperception (re)naissante du même pouvoir qui caractérise et annonce la personnalité complète et le premier fait de conscience. Biran explique le passage par le sentiment du pouvoir car nous n'avons aucun moyen de reproduire et donc de vouloir des sensations dépourvues d'effort. Le mode qui sert d'intermédiaire entre la contractilité purement animale (opérée par la réaction du centre organique) et la contractilité volontaire est celui de la contractilité spontanée qui s'effectue par l'action commencée dans le centre sur lequel la force s'exerce immédiatement et s'approprie par là à cette force, qui ne peut lui demeurer étrangère. L'autre exemple biranien est celui du passage des vagissements instinctifs de l'enfant aux mouvements spontanés (les habitudes contractées par le centre moteur) et enfin voulus.

Il y a par conséquent des niveaux de vigilance ou de veille, selon que ces modes sont ou non révocables, et le sommeil s'il signe la disparition du moi, ne signe pas, loin s'en faut, la disparition de toute activité ${ }^{34}$ :

Le principe qui entretient la vie et l'affectibilité dans les organes veille sans cesse, active excubias agit (Stahl), il parcourt, ensemble ou successivement, et dans un ordre déterminé par la nature ou les habitudes, toutes les parties de son domaine qui s'éveillent ainsi ou s'endorment tour à tour ; mais l'animal peut être assoupi pendant que plusieurs organes sont éveillés ; l'animal peut s'éveiller aussi pendant que la pensée et le moi s'éveillent encore ;

\footnotetext{
${ }^{32}$ Cf. Biran, 2001, p. 240, pour la définition de l'identité personnelle. Et Biran, 2001, p. 176 : «Changez en effet le terme organique résistant au sujet de l'effort, il n'y a plus d'identité reconnue; changez le sujet en laissant subsister le même terme, il n'y en aura pas non plus ».

${ }^{33}$ Biran, 2001, note p. 134-135.

${ }^{34} «$ Il n’y a de sommeil complet pour l'être sensitif que dans la mort absolue ». Biran, 1988, p. 94.
} 
il ne serait pas impossible d'observer ces gradations, ni peut-être, en les rapportant à leurs causes organiques, d'expliquer ainsi une partie des effets si surprenants du somnambulisme ${ }^{35}$.

Dormir c'est vivre, comme l'idiot, le maniaque, ou le dément, mais d'une vie toute sensitive :

Les deux éléments qui constituent l'homme double, sont si intimement unis dans son état naturel, que la réflexion a bien de la peine à les concevoir séparés ; mais dans les cas dont nous parlons, on peut les surprendre, les signaler à part. On les voit se succéder, s'exclure, se joindre, prédominer tour à tour, et former, sous la même enveloppe, deux êtres qui n'ont presque point de correspondance, ou sont aussi étrangers l'un a l'autre, que l'est l'homme éveillé aux actes qu'il a faits dans le somnambulisme. ${ }^{36}$

L'alternance de la veille et du sommeil illustre la dualité de l'homme (et non pas la dualité de conscience) : simplex in vitalitate, duplex in humanitate, selon la formule que Biran emprunte à H. Boerhaave. Mais Biran souligne en outre l'étrangeté des deux « individus » que composent notre veille et notre sommeil, l'étrangeté de la conscience vigile pour l'individu qui dort. L'étonnant alors n'est pas d'oublier, mais bien de nous rappeler nos rêves ${ }^{37}$, car s'il y a de la vie et des facultés toutes passives dans le sommeil, il n'y a pourtant aucun sentiment de soi.

Voilà en effet un état - la veille- où nous vivons avec la conscience de nos actes et de nos impressions, et l'autre - le sommeil - dans lequel « nous vivons sentons et agissons souvent par les mêmes organes, et en apparence de la même manière, mais sans conscience, sans moi, sans souvenir, et en demeurant comme étrangers dans l'un de ces états ou modes d'existence, à tout ce que nous avons éprouvé, senti, imaginé ou fait dans l'autre $»^{38}$. Et pourtant le moi, après s'être perdu, se retrouve au réveil dès qu'il recommence à vouloir. S'agissant de la question des causes physiologiques d'une telle suspension momentanée du vouloir, Bichat l'a examinée sous l'angle des deux vies, mais Biran entend corriger certaines inexactitudes. Certes les fonctions de la vie ont leurs périodes d'activité et de rémittence, et, par conséquent, le sommeil ne tient pas à l'absence activité

\footnotetext{
${ }^{35}$ Ibid.

${ }^{36}$ Ibid, p. 389.

37 Biran l'explique par le fait qu'une circonstance d'un songe par hasard tombe dans le cercle des images de la veille et réveille à sa suite les idées associées du songe sans qu'il y ait de rappel volontaire : par exclusion du souvenir plutôt que par souvenir proprement dit.

${ }^{38}$ «Si nous pouvions nous étonner des phénomènes que l'habitude nous a rendus les plus familiers, comment n'éprouverions-nous pas une surprise mêlée d'effroi en réfléchissant à cette différence si prodigieuse entre deux modes alternatifs d'existence, dans l'un desquels nous vivons, sentons et agissons, avec la conscience ou le sentiment intime de notre existence, de nos impressions et de nos actes, tandis que dans l'autre nous vivons, sentons et agissons souvent par les mêmes organes, et en apparence de la même manière, sans conscience, sans moi, sans souvenir, et en demeurant comme étrangers dans l'un de ces états ou modes d'existence, à tout ce que nous avons éprouvé, senti, imaginé ou fait dans l'autre ? Sans doute le phénomène du sommeil a dû faire naître de très bonne heure dans l'esprit de l'homme, avec l'idée de la mort ou d'un sommeil éternel, celle d'une survivance ou d'une vie qui ne fait que changer de forme quand elle semble cesser d'être ». (Biran, 1984, p. 82.
} 
des organes : les sens et l'imagination s'exercent dans le sommeil aussi. S'il n'y pas de sommeil partiel (c'est-à-dire de suspension seulement partielle du sentiment de soi), en revanche l'imagination joue tout son rôle dans le sommeil comme faculté de produire spontanément des images ou intuitions simples: de telles images et de tels «actes», hors de portée du souvenir, forment « une portion d'existence tout à fait séparée ou détachée de la personne ». ${ }^{39}$

Biran s'appuie aussi sur les analyses de Barthez et de Cabanis dont il veut réunir les vues pour ce qui concerne la physiologie du sommeil. Pour Barthez, il y a concentration du principe de vie et de la sensibilité dans les organes qui à l'état de veille sont en solidarité sympathique, et un ralentissement des fonctions intérieures; selon Biran, la concentration va de pair avec le surcroit d'activité si l'on distingue les forces «radicales» (Barthez) qui augmentent (exemple de la digestion accélérée après le repas dans le sommeil, ou de l'hibernation de certains animaux) et celles, effectives, qui tombent au-dessous du ton de l'état de veille. Ainsi l'âme est confusément affectée dans le sommeil par les résultats de l'action de la force vitale.

Quand la force se déploie sur l'ensemble des organes qui lui sont soumis, le moi est donc présent à lui-même tant que la veille dure. La suspension de l'effort qu'est le sommeil suppose par conséquent des causes qui interrompent l'effort soit en se portant sur l'organe immédiat par lequel la force s'exerce, soit sur des parties mobiles subordonnées à l'action, soit encore en interceptant les communications directes qui lient la force et les organes mobiles. En d'autres termes, la cause du sommeil, selon Biran, ne saurait partir immédiatement de la vie organique qui n'est pas affectée par la suspension de l'effort sinon de manière indirecte. Parmi les causes du sommeil susceptibles d'empêcher l'action continuée de la volonté, il y en a de passives: l'influence délétère de substances narcotiques, les somnifères qui ôtent au centre moteur son empire et aux organes mobiles leur moyen d'obéissance, l'opium, le vin... le corps alors n'est plus soutenu par la puissance dont l'exercice fait l'état de veille. Même la cause qui mène au sommeil complet produit, dans un degré moindre, l'exaltation ou le délire qui se caractérisent par la suspension de la faculté volontaire (exemple des maladies ou des fièvres dans lesquelles les muscles ne se contractent plus volontairement). Si l'alternance entre la veille et le sommeil a la nécessité d'une loi de la nature, « de même que celle des jours et des nuits auxquels elle est liée » ${ }^{40}$, l'intervalle qui les sépare, souligne Biran, est plutôt, elle, une loi de l'habitude.

Mais le sommeil est une fonction active quant à son principe ${ }^{41}$ : comment est-ce possible si la force est «hyper-organique»? Biran répond que la force, pour être hyper-organique, est enchaînée aux lois des organes sur lesquels elle agit, et son exercice par conséquent conditionné : il doit exister des liens de communication directe entre le centre et les organes intègres et la réaction de l'organe mobile doit être proportionnée à la force déployée ; sans le sentiment de l'existence de la partie mobile, la volonté ne pourrait naitre. Ainsi l'hémiplégie est un véritable sommeil des parties paralysées, lesquelles mènent une vie « absolue » qui ne répond plus à l'effort propre à la vie

\footnotetext{
${ }^{39}$ Ibid, p. 85.

${ }^{40}$ Ibid, p. 89.

${ }^{41}$ Ibid.
} 
de relation; de même l'affectibilité pure est le sommeil de l'effort. La cause du sommeil dans tous les cas consiste dans l'oppression ou la suspension momentanée de l'action de la volonté sur les organes qui lui sont soumis. ${ }^{42}$

Mais l'effort d'une veille prolongée entraine nécessairement la fatigue, et Biran rend compte de la qualité de nos sommeils nocturnes ou diurnes selon que l'activité des organes du mouvement et de ceux des sens ou de l'imagination y ont été diversement mobilisés. Ainsi un surcroît d'imagination non seulement n'entraîne pas le sommeil, mais peut même l'empêcher; la volonté cherche alors elle même les moyens du repos et dirige son activité contre le principe de cette activité même :

Elle ferme d'abord les sens dont elle dispose aux impressions qui pourraient encore les provoquer; elle place le corps dans une attitude telle qu'il n'ait plus besoin d'effort pour être soutenu, et que les muscles relâchés n'aient plus à supporter, comme dans la veille, le travail de la station ou de l'assiette fixe du corps. Enfin la volonté suspendant toutes les fonctions qui sont de son ressort, et travaillant pour ainsi dire à se rendre inutile ou nulle, amène le sommeil en reproduisant par son absence la condition essentielle à laquelle il se lie. ${ }^{43}$

Travailler à se rendre nulle, voilà en effet pour la volonté une tâche aussi nécessaire que contradictoire en apparence ! Et si nous dormons comme nous veillons, selon que la volonté est ou non exercée intensément à l'état de veille, la lassitude qui suit dans les organes du mouvement provoquera le besoin de sommeil, notamment si l'exercice seul de la pensée a rempli notre veille. Mais tout effort prolongé amène le sommeil lié à l'affaiblissement progressif de l'effort et enfin à sa nullité complète. Ainsi la concentration des forces motrices dans les organes exercés accroit en effet leur irritabilité et les rend indépendants « de l'action du centre moteur ou de la direction de la volonté » :

Lorsque nous sommes fatigués par une marche forcée, nous sentons très bien que nos jambes continuent d'elles-mêmes les mouvements si souvent répétés, et nous portent, comme on dit, machinalement, sans qu'il y ait presque aucun effort de la volonté ; il n'y a pas d'état plus voisin du sommeil que celui-là. Si le marcheur, disposé de cette manière, s'arrête un moment, il s'endort; et on en a vu souvent dormir même en marchant. ${ }^{44}$

Parce que certains mouvements s'associent avec le sommeil (tel est le cas du somnambulisme), il faut distinguer des mouvements machinaux (ainsi des soubresauts convulsifs qui précèdent le sommeil complet) les mouvements volontaires conscients.

Retenons que le surcroit d'activité des organes intérieurs pendant le sommeil - qui est l'inaction des sens externes -, fait prédominer les affections immédiates, dont l'individu par conséquent n'a nulle conscience :

L'être sensitif qui pâtit ou qui jouit est déterminé à divers actes ou mouvements très coordonnés qui se proportionnent nécessairement à la nature des affections qu'il éprouve, et

\footnotetext{
${ }^{42}$ Pour l'examen de ces modalités, se rapporter au même endroit.

${ }^{43}$ Biran, 1984, p. 90.

${ }^{44}$ Ibid, p. 92.
} 
sont pour l'observateur étranger des signes de ces dernières, pendant que le moi ou la personne absente ignore complètement tout ce qui se passe dans l'organisation. ${ }^{45}$

Une fois ôtée la puissance d'effort, reste seulement la faculté passive de sentir, de recevoir des impressions et d'en être affecté. Les facultés en exercice dans le sommeil sont aussi celles par exemple de l'homme passionné à l'état de veille, de rêve éveillé (songe) ou de somnambulisme ; à l'état de veille, même le plus complet, s'exerce la sensibilité intérieure avec laquelle l'imagination a partie liée :

Il n'y a qu'a détourner l'attention des objets externes, cesser de réagir sur soi-même et se laisser aller à cet état qu'on appelle rêverie, et qui est plus marqué surtout dans certaines dispositions de la sensibilité, pour reconnaître tout l'ascendant de cette puissance aveugle et qui s'ignore complètement, lors même que ses inspirations sont les plus heureuses. ${ }^{46}$

Notre vigilance n'est jamais telle que nous ne puissions nous laisser aller à la rêverie, et gagner par les mouvements des passions ou de l'imagination, et certaines dispositions de la sensibilité. Biran explique ainsi le «sentiment confus d'un mode d'existence antérieur» par le retour périodique des mêmes images, qu'il s'agisse du rajeunissement de l'imagination des vieillards revivant les tableaux du premier âge, ou de la façon dont nous sommes parfois frappés par certains tableaux dont nous ne savons s'il s'agit du retour de certaines images confuses " qui s'enfoncent pour ainsi dire dans les profondeurs du temps, et se perdent dans le vague de l'existence passée $\gg .{ }^{47}$ L'imagination dans tous les cas réalise ce qu'elle conçoit, et exclut l'attention, et l'on peut dire de la vie de ceux qui ont beaucoup d'imagination qu'elle n'est guère autre chose qu'un « rêve continuel $»{ }^{48}$.

\section{Somnambuler}

Biran connaît et lit J.-P.-F. Deleuze, assiste à des séances d'expériences magnétiques. Et le Docteur J.F. Delpit, Secrétaire de la Société Médicale de Bergerac fondée par le philosophe, lui fournit aussi matière à penser le somnambulisme magnétique. Dans le somnambulisme, la communication sympathique du cerveau avec les organes de la locomotion et de la voix comme dans la veille est entière ou même accrue. Le somnambule a l'intuition des objets présents aux sens externes quoi que ceux-ci soient fermés en partie, et il exerce les mouvements nécessaires pour aller vers eux ; bref, il se comporte comme il pourrait le faire à l'état de veille ! « Tout trompe dans le somnambule! » ${ }^{49}$, s'exclame Biran. Car ce dernier agit en effet comme si son action était éclairée, intelligente et volontaire alors qu'il est actif en résultat mais non point en principe, puisqu'il...dort.

Tout se passe comme si son être semblait se diviser en deux personnes distinctes :

\footnotetext{
${ }^{45}$ Biran, 1988, p. 93.

${ }^{46}$ Ibid, p. 95.

${ }^{47}$ Biran, 1984, p. 96.

${ }^{48}$ Ibid, p. 98.

${ }^{49}$ Ibid, p. 120.
} 
En effet, comme tout mouvement spontané, ou qui a son principe caché dans l'intérieur même de l'être qui le manifeste, est pour nous le signe ou le caractère essentiel de la vie ; ainsi toute série régulière de ces mouvements, ordonnés entre eux et appropriés à un but, paraît être le caractère essentiel d'une pensée, d'une volonté ; car nous ne pouvons voir que les mouvements extérieurs, et le principe de l'action comme les ressorts auxquels il s'applique, échappent par leur nature aux sens et à toute faculté de représentation. Mais si, d'une part, c'est une volonté, une intelligence parfaitement éclairée qui préside aux phénomènes du somnambulisme, pendant que, d'autre part, les faits les mieux constatés ne permettent pas de douter qu'alors le sommeil ne soit complet et absolu, quel est le caractère ou signe naturel qui servira à distinguer deux états ou modes d'existence, si différents et si parfaitement étrangers l'un à l'autre, qu'en s'appliquant tour à tour à un seule et même être vivant, ils semblent diviser cet être en deux personnes distinctes, dont l'une ne s'approprie rien de ce que l'autre a fait ou senti, n'en conserve pas le moindre souvenir, n'y joint pas le même moi $?^{50}$

Puisqu'en aucun cas la volonté ne peut diriger les actes du somnambule, c'est l'imagination qui doit présider à tout, en retraçant un objet dans le sens interne sans se régler sur aucune perception externe : l'imagination réalise au dehors, souligne Biran, ce qu'elle crée au dedans. Voilà donc un être qui dort profondément et se conduit avec un soin et une vigilance extrême ! Biran prend l'exemple de l'abbé du séminaire de Bordeaux :

[II] se levait chaque nuit, se mettait à son bureau, écrivait des sermons très suivis dans toutes leurs parties, en se corrigeant, faisant des ratures et substituant au mot raturé un autre mot qu'il plaçait exactement au-dessus. D'autres fois il copiait de la musique, après avoir rayé son papier avec une canne; il observait parfaitement la valeur des notes, et la place des paroles correspondantes qu'il écrivait au-dessous. Pour s'assurer s'il s'aidait en quelque chose de l'organe extérieur de la vue, on mit un corps opaque devant ses yeux, pendant qu'il notait sa musique ; mais il n'en fut point empêché, et continua son travail comme auparavant. On essaya encore de substituer un autre papier à celui sur lequel il écrivait; mais il parut sentir la différence, et rejeta ce nouveau papier, jusqu'à ce qu'on lui eût substitué une feuille absolument égale en dimension à celle dont il se servait; alors il continua en reprenant à la même place où son carton finissait, sur sa feuille, sans paraître apercevoir que la nouvelle était toute blanche; ce qui prouve qu'il se dirigeait alors par le toucher joint à l'imagination et non par la vue. Voilà des songes intellectuels, joints à l'emploi des signes et à l'usage de la locomotion, qui ne semblent mettre aucune différence sensible entre l'état de ce somnambule et celui de la veille la plus complète. Cependant la différence est entière, même dans ce cas, etc. ${ }^{51}$

L'individu ne veillant pas, il n'y a aucun souvenir des actes du somnambule de la part de l'homme éveillé : on a affaire à deux principes d'action, sinon à deux personnes distinctes; chez le somnambule l'exercice des sens est entièrement guidé par l'imagination, ses mouvements

\footnotetext{
${ }^{50}$ Ibid, p. 113.

${ }^{51}$ Biran puise ces anecdotes dans l'Encyclopédie ou Dictionnaire raisonné des sciences, des arts et des métiers, à l'article « somnambulisme » et dans le Dictionnaire des merveilles de la nature, de Brisson. (Biran, 1984, p. 116).
} 
coordonnés et extraordinairement précis sont impassibles, automatiques, mais rendus possibles par la sympathie des muscles locomoteurs avec le centre cérébral. Conformément aux analyses de son premier Mémoire, Biran souligne combien l'habitude facilite les mouvements (y prêter attention c'est les rater) dont l'aisance est ici proportionnelle à leur aveuglement (aisance à laquelle il faut ajouter la communication sympathique entre les muscles et le cerveau comme organe de l'intuition). Ainsi le somnambule peut voir et entendre mieux que l'homme éveillé.

Biran en tire cependant une étonnante conclusion sous la forme, il est vrai, d'hypothèse :

Il n'est pas impossible que ce soit la même substance, la même force qui agisse alternativement, tantôt sur le système musculaire de la vie animale, avec la conscience de son effort, ce qui constitue le moi permanent dans l'état de veille, et tantôt sur le système musculaire de la vie organique qui constitue un autre moi ayant un autre système d'idées tout à fait différentes et relatives à ce qui se passe dans l'intérieur du corps. Comme dans la veille le moi ignore ce qui se passe dans l'organisme, et tout cet ordre d'affections qui se rapportent aux fonctions vitales, de même pendant le sommeil, cet autre moi qui agit dans l'intérieur est étranger aux impressions de la vie animale et aux mouvements qui s'y rapportent.

Il paraîtrait que le sommeil magnétique consiste non seulement, comme le sommeil ordinaire, dans une suspension de l'action de l'âme sur le système musculaire de la vie animale, et en général sur tous les organes de cette vie, mais de plus dans un mode d'action particulière qu'elle acquerrait sur le système musculaire et sur l'ensemble des organes de la vie intérieure. De là peut-être la possibilité d'expliquer plusieurs phénomènes extraordinaires du somnambulisme magnétique. ${ }^{52}$

Affirmer qu'il y a deux moi en une seule et même personne vivante, est une hypothèse bien surprenante de la part d'un philosophe qui nous explique que le moi est un et ne se divise pas ! Elle est pourtant plus plausible que celle d'un même moi tantôt de la veille, tantôt du sommeil...Mais le sommeil magnétique est-il réellement la veille d'un autre moi ? Dans la veille, l'on perçoit (vie animale) et l'on sent (vie intérieure), ou plutôt la sensation est à la fois sentie et perçue, mais ce qui est senti n'a pas besoin en tant que tel d'être perçu. Le somnambule peut donc être affecté sans percevoir. Mais le somnambule et l'homme éveillé sont deux personnes différentes dont chacune ce qui est impossible dans le sommeil ordinaire - tire parti des connaissances de l'autre, et « l'entend ou se souvient de ce qu'elle a dit ou fait, sans néanmoins s'identifier avec elle ${ }^{53}$. Si l'on peut faire l'hypothèse d'une identité de substance, on ne peut faire celle d'une identité de personne. Qu'on nous permette de citer ce long passage :

On a vu des somnambules parler d'eux-mêmes comme si leur individu dans l'état de veille était une personne toute différente de leur individu dans l'état de somnambulisme.

Une demoiselle Adélaïde n'avait aucune idée du moi proprement dit. Elle ne convenait jamais de l'identité d'Ad. Avec Petite, nom qu'elle se donnait pendant sa manie.

\footnotetext{
${ }^{52}$ Biran, 1990, p. 355. Nous soulignons.

${ }^{53}$ Ibid, p. 357.
} 
Mlle N, qui, après avoir perdu sa fortune, se détermina à entrer au théâtre, condamnait ce projet dans des accès de somnambulisme, et lorsqu'on lui demandait pourquoi elle y tenait, elle répondait : « ce n'est pas moi, c'est elle », «pourquoi ne l'en détournez-vous pas ? », « que voulez-vous que je lui dise, c'est une folle». Voilà deux personnes qui se connaissent, se communiquent, savent réciproquement ce qu'elles pensent, n'en sont pas moins deux personnes séparées : et si l'on dit que c'est la même substance âme qui constitue les deux personnes, ou les réunit, je ne vois plus d'absurdité à dire comme Spinoza, qu'il n'y a qu'une seule substance pensante, dont toutes les pensées individuelles séparées ne sont que des modes ou des attributs. En effet d'après les exemples cités, il n'y a pas plus de distinction entre Pierre ou Jean, et moi, qu'il n'y en a entre le moi de la veille et celui du somnambulisme, et si ces deux moi se réunissent dans la même substance, comme en étant des modifications distinctes, je ne vois pas pourquoi tous les êtres pensants qui se reconnaissent chacun comme des personnes ou des individualités séparées, ne seraient pas également des modes d'une substance unique.

Il est remarquable que le moi du somnambule connaisse l'autre et le juge, comme il jugerait une personne étrangère, tandis que le moi de la veille n'a aucune idée de celui du somnambule, qui lui reste toujours inconnu; c'est là une différence totale avec ce qui arrive dans l'état ordinaire, où le moi juge les affections et les passions qui sont sous l'empire d'une vie sensitive, tandis que le principe qui dirige les mouvements de celle-ci, ignore complètement ce qui se passe dans l'autre, et reste étranger à ses idées.

Cette observation tendrait à faire croire que lorsque l'âme change d'instrument, de centre ou de mobile d'action, en déployant sur les organes de la vie intérieure une activité semblable à celle qu'elle déploie ordinairement sur les muscles volontaires et les organes de la vie intérieure, elle voit encore passivement les traces des impressions conservées dans le cerveau, mais n'ayant aucun sentiment actuel, ni à plus forte raison aucun souvenir de son action sur le cerveau, ces traces doivent lui paraître aussi étrangères que si elles les recevaient d'ailleurs, et c'est comme si elle lisait dans un livre, ou entendait les discours d'une personne étrangère, ou se rappelait avoir lu ou entendu, etc. Il arrive à peu près la même chose dans le sommeil ordinaire, lorsqu'on croit entendre des discours ou des vers que le sens interne de l'imagination compose lui-même; dans cet état, l'âme n'agissant pas sur le centre où ces images sont produites, en reçoit l'impression comme si elle venait du dehors car elle ne peut s'attribuer à elle-même ou à son effort ce qu'elle ne fait pas, et elle ne s'approprie réellement que les actes, mouvements, idées, dont elle est cause. Mais l'âme peut se souvenir au réveil de ces impressions conçues dans l'état de sommeil ordinaire parce qu'elle réagissait sur le même organe, sans être déviée de ses lois ordinaires. ${ }^{54}$

\section{Conclusion}

\footnotetext{
${ }^{54}$ Ibid, p. 358.
} 
Entre la veille, le sommeil, et l'état magnétique ou somnambulique, il y a des plans d'existence qui sont « autant de manières de veiller ou de dormir ». Si la folie est un rêve continuel dont on ne s'éveille pas, la veille signe la présence active de l'effort immanent. Pourtant cette veille sans intentionnalité est aussi la source de toutes les distractions, diversions et aliénations possibles. Comme lorsque l'âme ne lit plus en elle-même que comme dans un livre, parce que les traces affectives et intuitives de sa vie sensitive ne peuvent donner lieu à aucun sentiment d'action. Comme dans le somnambulisme magnétique où se réalise la soumission - non pas de la volonté mais du désir au désir sympathique du magnétiseur. Et Biran constate ce fait jusque dans l'état de veille :

Deux personnes qui sont à l'unisson de sensibilité, ou qui sympathisent complètement entre elles, ont une manière de s'entendre, ou de se sentir réciproquement sans se voir, ni se parler, ni se toucher, alors même qu'elles se regardent et se parlent ${ }^{55}$.

L'injonction biranienne (penser à être soi !) s'est nourrie sans conteste de la hantise de n'être pour rien dans ce que l'on est : elle est à la mesure de cette écoute forcenée de soi, déployée dans l'effort de réflexion proprement philosophique (fondé sur le sens de l'ouïe couplé à la voix), redoublement ou repliement de la vigilance, qui tente incessamment de conjurer l'accidentelle absence à soi. Cette dernière semble pourtant la règle s'agissant de la vie mondaine ou sociale, par opposition à la vie solitaire réfléchie du penseur :

Je suis plus ou moins distrait, c'est-à-dire que je donne plus ou moins mon attention à chaque chose suivant mes dispositions organiques bonnes ou mauvaises [...] dans plusieurs cas je suis dans le monde comme un somnambule [...] c'est dans le cabinet et en présence de moimême que je suis moins distrait ou somnambuliste ${ }^{56}$

Dans son Journal, en mai 1817, Biran affirme qu'il assiste avec distraction aux séances de la chambre, « et comme dans le somnambulisme » ${ }^{57}$... Au même Journal il confie aussi sa timidité, et par conséquent sa grande difficulté à prendre la parole en public, à la Chambre.

Doit-on s'étonner qu'un grand lecteur de Biran, Gabriel Tarde, ait fait de la vie sociale ellemême un rêve magnétique fondée sur une vie toute sympathique, de croyance et de désir ? La timidité est en effet, si l'on en croit Tarde, une «magnétisation consciente, et par suite incomplète, comparable à cette demi-somnolence qui précède le sommeil profond où le somnambule parle et se meut ${ }^{58}$.

A celui qui affirme «être dans le monde comme un somnambule » ${ }^{59}$, une telle filiation n'aurait certes pas déplu : la société ne fut-elle pas le sommeil magnétique de Biran, et le moi social sa « folie » propre?

\footnotetext{
${ }^{55}$ Ibid, p. 355.

${ }^{56}$ Maine de Biran, 1955, p. 21.

${ }^{57}$ Maine de Biran, 1955, p. 45.

${ }^{58}$ Gabriel Tarde, 1921, p. 93.

59 « J'ai dit que j'étais comme somnambule dans le monde extérieur, des affaires etc. Il y a en effet bien des manières de dormir, et tout autant qu'il y a des manières de veiller; la sensibilité peut être toute retirée en dedans pendant que
} 
Anne Devarieux, Normandie Université, France; Identité et subjectivité, F-14032 Caen, France ; EA 2129. annedevarieux@wanadoo.fr

\section{Bibliographie}

Azouvi, François (2000). Maine de Biran. La science de l'homme. Paris : Vrin.

Devarieux, Anne (2004). Maine de Biran l'individualité persévérante. Grenoble : Millon.

Henry, Michel (1965) Philosophie et phénoménologie du corps. Essai sur l'ontologie biranienne. Paris : PUF.

Maine de Biran (1954-1955-1957). Journal. 3 vol., Neufchâtel : La Baconnière.

Maine de Biran (1984). Discours à la société médicale de Bergerac. Paris : Vrin.

Maine de Biran (1987). Influence de l'habitude sur la faculté de pensée. Paris : Vrin.

Maine de Biran(1988). Mémoire sur la décomposition de la pensée. Paris : Vrin.

Maine de Biran (1990). Commentaires et marginalia XIXème. Paris : Vrin.

Maine de Biran (1993). Commentaires et marginalia XVIIIème. Paris : Vrin.

Maine de Biran (1995). De l'aperception immédiate. Paris : Vrin.

Maine de Biran (1996). Correspondance philosophique. Paris : Vrin

Maine de Biran (2001) Essai sur les fondements de la psychologie et sur ses rapports avec l'étude de la nature. Paris : Vrin.

Gabriel Tarde (1921) Les lois de l'imitation. Paris : Alcan.

Xavier Tilliette, (1983). Nouvelles réflexions sur le cogito biranien. Revue de métaphysique et de morale, $\mathrm{n}^{\circ} 4$, p. 436-446.

\section{LA VEILLE DU MOI OU L'EXISTENCE VIGILE}

l'activité se porte au dehors. Au contraire l'activité peut se concentrer au dedans et dans l'exercice le plus intime de la pensée pendant que la sensibilité est occupée au dehors » (Maine de Biran, 1955, p. 422). 
Égalité de l'effort (force hyper-organique et résistance du corps propre)

Identité et unité de la personne, de sa vie de relation.

Les deux modalités de l'effort (variation intensive)

Effort inintentionné ou immanent.

Effort sans effort apparent de la volonté

tension du corps en masse

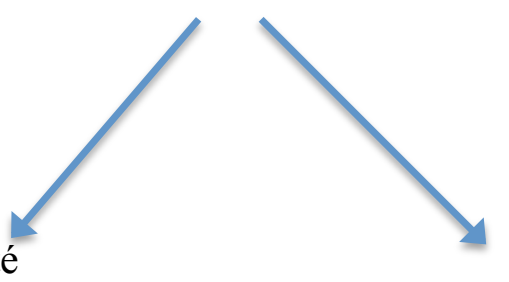

Effort intentionné ou exprès

Représentation d'objets.

Activation expresse des sens

(vision, toucher, etc.

Vigilance sans objet

Moi "pur” ou moi "phénoménal“" (sentinelle de Stahl)

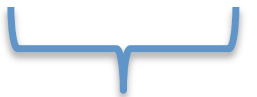

Aperception

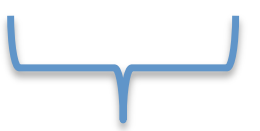

Perception. Attention. Représentation

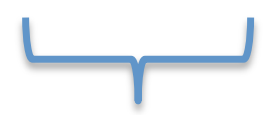

Réflexion : repliement de l'individu sur ses propres actes (la voix et l'ouïe).

Redoublement de la vigilance.

États du corps, états du moi biranien. Tableau .

\begin{tabular}{|l|l|l|}
\hline Veille & Sommeil & Somnambulisme \\
\hline $\begin{array}{l}\text { Effort immanent fondé sur la } \\
\text { dualité intérieure du fait } \\
\text { primitif de conscience : force } \\
\text { hyper-organique et } \\
\text { résistance intérieure du corps }\end{array}$ & $\begin{array}{l}\text { Suspension momentanée du } \\
\text { vouloir, de l'effort, de la } \\
\text { conscience. Activité vitale } \\
\text { non consciente d'elle-même. }\end{array}$ & $\begin{array}{l}\text { Etat intermédiaire entre le } \\
\text { sommeil profond sans rêve } \\
\text { et la veille. Suspension } \\
\text { momentanée du vouloir, de } \\
\text { l'effort. Activité vitale non } \\
\text { consciente d'elle-même. }\end{array}$ \\
\hline $\begin{array}{l}\text { Présence du moi (sentiment } \\
\text { /idée) }\end{array}$ & $\begin{array}{l}\text { Disparition du sentiment de } \\
\text { soi. Absence de jugement, } \\
\text { d'attention }\end{array}$ & $\begin{array}{l}\text { Suspension du sentiment du } \\
\text { moi }\end{array}$ \\
\hline
\end{tabular}




\begin{tabular}{|c|c|c|}
\hline $\begin{array}{l}\text { Tension générale du corps } \\
\text { résistant ; sentiment des } \\
\text { parties mobiles }\end{array}$ & $\begin{array}{l}\text { Absence de résistance du } \\
\text { corps }\end{array}$ & \\
\hline Mouvements volontaires & $\begin{array}{l}\text { Mouvements instinctifs et } \\
\text { spontanés, machinaux }\end{array}$ & $\begin{array}{l}\text { Sympathie des muscles } \\
\text { locomoteurs et du centre } \\
\text { cérébral. Motilité animale. }\end{array}$ \\
\hline \multirow[t]{2}{*}{$\begin{array}{l}\text { Vie animale ou vie de } \\
\text { relation }\end{array}$} & $\begin{array}{l}\text { Vie organique, purement } \\
\text { sensitive ou vie absolue. } \\
\text { Affections. Appétits et } \\
\text { penchants. }\end{array}$ & \\
\hline & $\begin{array}{l}\text { Surcroit d'activité des } \\
\text { organes internes } \\
\text { Exercice de l'imagination ou } \\
\text { de l'intuition spontanée } \\
\text { passive }\end{array}$ & $\begin{array}{l}\text { Absence de perception. } \\
\text { Intuition dans le sens interne. } \\
\text { Affection. Absence de } \\
\text { souvenir. }\end{array}$ \\
\hline $\begin{array}{l}\text { Hypothèse : la force agit } \\
\text { sur le système musculaire } \\
\text { de la vie animale }\end{array}$ & & $\begin{array}{l}\text { Hypothèse : la force agit } \\
\text { sur le système musculaire } \\
\text { de la vie organique }\end{array}$ \\
\hline
\end{tabular}

